\title{
Media Modeling for Distillation Process with Modelica/MWorks
}

\author{
Chen Chang Ding Jianwan Chen Liping Wu Yizhong \\ CAD Center, Huazhong University of Science and Technology, Wuhan, China, 430074 \\ chenchang1210@hotmail.com jwdingwh@gmail.com chenlp@hustcad.com \\ cad.wyz@hust.edu.cn
}

\begin{abstract}
This paper describes a package which consists of media models for distillation process with the method of classical two-parameter cubic thermodynamic equation of state in Modelica on platform MWorks. A structuralized and hierarchical modeling strategy was proposed in order to separate the components and the mixing rules of mixture from the vapor-liquid equilibrium predicting methods. With this strategy, a new media model can be introduced by changing the components' information of mixture and of mixing rule. An air distillation column model was built to test the air media model consists of nitrogen, oxygen and argon. The results obtained with MWorks were compared with simulation calculated with Aspen Plus. A very good agreement was found and the air model for distillation in the column worked well.
\end{abstract}

Keywords: media model; distillation; Modelica; Vapor-Liquid equilibrium

\section{Introduction}

Distillation process utilizes the different boiling point of components of mixture on the same condition to separate components from mixture. It is widely used in petroleum refining, chemical, metallurgical industries and so on. Up to the present there are numerous commercialized platforms like Aspen Plus and HYSYS that could be used to predict distillation process. These platforms mainly contain media model for vapor-liquid equilibrium (VLE), physicochemical constants database, process unit models, User Interface and algorithms library. Therein the media model is one of the most important cores of these tools. Its computational efficiency and accuracy have a strong impact on the simulation consumed time and accuracy of the whole process system model. The prediction methods of vapor-Liquid equilibrium in these tools are mainly fugacity coefficient method and fugacity- activity coefficient method. With the former method the fugacity coefficients of vapor phase and liquid phase are calculated from the thermodynamic equation of state. The fugacity coefficient of vapor is from the thermodynamic equation of state (EoS) and the activity coefficient from excess function with the later method. The users of these tools just know how to use vapor-liquid equilibrium model but don't know the mechanism of modeling in these tools.

At present, there are lots of media models for pure substance and mixture in the Modelica Standard Library (MSL) Media[1]. But there is no media model for vapor-liquid equilibrium. In this paper, a package named MediaForSapera which contains physical property computing with the two-parameter EoS method for vapor-liquid equilibrium was built based on Modelica in MWorks[2]. The models in this package are based on general concept which allows the introduction of new working media for distillation by modification of a new model parameters and addition of particular equations of different EoS methods. The media package consists of parts as follows:

1、 Interfaces: consists of the partial media models that contain the common variables, constants, parameters and model structure. At present there is a base partial model and four partial models with two-parameter cubic EOS methods in the package.

2、 FluidData: consists of records contain the basic physicochemical constants, like molecular weight and critical temperature, and experimental regression coefficients of pure component of the mixture, such as coefficients of polynomial for specific enthalpy calculation.

3、 Examples: some mixture models for Vapor-Liquid Equilibrium, such as air model which is made up of nitrogen, oxygen and argon, simple air model composed of nitrogen and oxygen. All examples in the package are extends from package Interfaces. 
4. Tests: some simple models that are used to test the media models in Examples.

\section{Methods of VLE based on the two-parameter EoS}

Vapor-liquid equilibrium is a condition where a liquid and its vapor are in equilibrium with each other, a condition or state where the rate of evaporation equals the rate of condensation on a molecular level. It could be described by fugacity's equality of two phases as follow:

$$
f_{i}^{V}=f_{i}^{L}
$$

Where $f_{i}^{V}$ and $f_{i}^{L}$ means the fugacity in vapor and liquid phase of component $i$ respectively. There are two methods that are used to calculate the fugacity. One is the fugacity-activity coefficient method. Another is fugacity coefficient method which predicts the fugacity of two phases by EoS mean as follows:

$$
\left\{\begin{array}{l}
f_{i}^{V}=\Phi_{i}^{V} y_{i} p \\
f_{i}^{L}=\Phi_{i}^{L} x_{i} p
\end{array}\right.
$$

Where $\Phi_{i}^{V}$ and $\Phi_{i}^{L}$ means the fugacity coefficient in vapor and liquid phase of component $i$ respectively and $y_{i}$ and $x_{i}$ the mole fraction in vapor and liquid phase of component $i$ respectively.

The fugacity coefficient method has good thermodynamic consistency and doesn't need the fugacity of standard state when it is calculated. Its results agree with the experimental measurements very well for a range of mixture except the strong polar molecule and electrolyte system. So it is widely applied to the prediction of vapor-liquid equilibrium of mixture. The two-parameter cubic EoS method is introduced into this paper to model VLE of mixture.

\subsection{Two-parameter EoS}

The thermodynamic equation of state has been proposed which has practical utility for the first time by Van der Waals in year 1873 and it has been developed rapidly in the next one hundred years. The EoS has been widely used in VLE calculation in large range of mixture, even includes strong polar molecule, during the latest decades. Herein the two-parameter EoS, such as Soave-Redlich-Kwong (SRK) equation, Redlich-Kwong equation (RK), Peng-Robinson (PR) equation and Harmens equation, has characteristic of a small number of parameter, simple expressions, easily-solving and exact results. So it has been developed very well and applied more widely than other EoS.

In this paper, four kinds of two-parameter cubic EoS were introduced to model the VEL of mixture. They are RK, SRK, PR and Harmens and they could be expressed in a unified form:

$$
p=\frac{R T}{v-b}-\frac{a(T)}{v^{2}+m b v+n b^{2}}
$$

Where, $p$ is the pressure of the media, $p a$. $T$ the temperature of the media, $K . v$ molar volume, $\mathrm{m}^{3}$ $/ \mathrm{mol} . R$ the universal gas constant, $8.314 \mathrm{~J} /(\mathrm{mol} \cdot \mathrm{K})$. $a(T)$ and $b$ the parameters of EoS, these two parameters of mixture are computed by mixing rule. $m, n$ different constants according the different EoS. The fugacity coefficient and specific enthalphy of mixture are the important medium properties for distillation process simulation. For two-parameter cubic EoS method, they are computed as follows:

$$
\begin{gathered}
R T \ln \phi_{i}=\int_{V}^{\infty}\left[\left(\frac{\partial p}{\partial n_{i}}\right)_{T, V, n_{j}}-\frac{R T}{v}\right] d v-R T \ln z \\
h_{i}=\int_{V}^{\infty}\left[p-T\left(\frac{\partial p}{\partial T}\right)_{V, n_{T}}\right] d V+p V+h_{i}^{0} \\
h=\sum y_{i} h_{i}
\end{gathered}
$$

where, $z$ means the compressibility factor, $=p v /(R T)$. $h_{i}$ the actual specific enthalphy of component $i$ of mixture. $h_{i}^{0}$ the ideal specific enthalphy of component $i$ of mixture. $h$ the actual specific enthalphy of mixture.

\section{2 mixing rule}

When the equation (3) is applied to mixture, the parameters $a(T)$ and $b$ should be computed by some mixing rules that could be found in reference[3]. For $a(T)$ and $b$ in RK, SRK and PR EoS, the Reid mixing rule is used usually.

$$
\left\{\begin{array}{c}
a_{m}=\sum_{i} \sum_{j} y_{i} y_{j} \sqrt{a_{i} a_{j}}\left(1-k_{i j}\right) \\
b_{m}=\sum_{i} y_{i} b_{i}
\end{array}\right.
$$

Where, $a_{m}$ and $b_{m}$ mean the parameters of EoS for mixture. $a_{i}, b_{i}$ and $a_{j}$ the parameters of components $i$ and $j . y_{i}$ and $y_{i}$ the mole fraction of components $i$ and $j$ in vapor or liquid phase of the mixture. $k_{i j}$ is binary interaction coefficient between component $i$ and component $j$. It can be obtained from experiment. The mixing rule for Harmens EoS is different from the other three EoS. It can be consulted in reference[4].

\section{Implement in MWorks}

There are large numbers of mixture in the distillation process industries and so are the media properties 
prediction methods and the mixing rules of introduction of new working media for distillation by components in mixture. In order to allow the modifying the minimizing set of parameters and

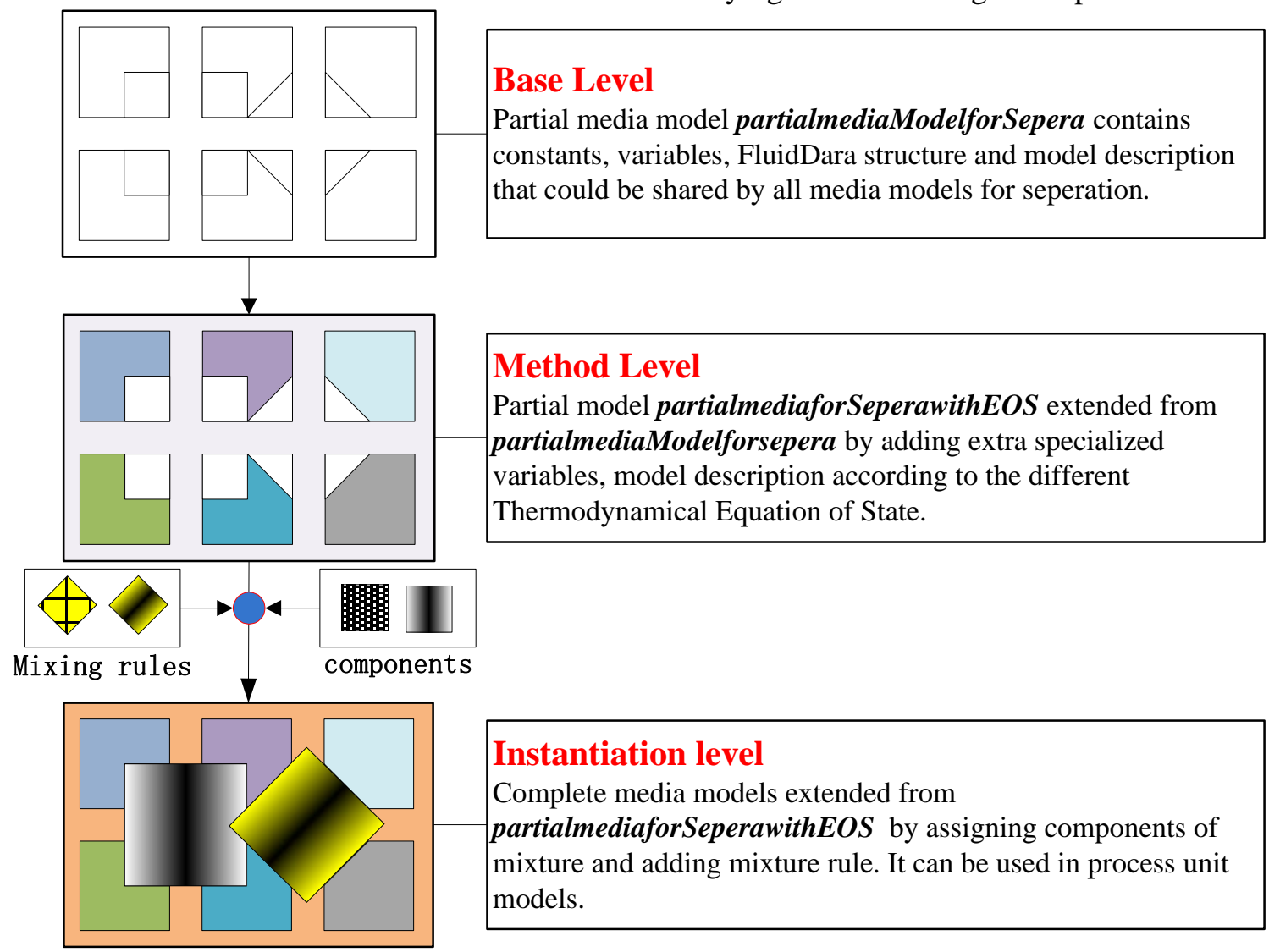

Figure 1 Sketch of modeling strategy of media model for distillation

adding the minimizing set of equations, a structuralized and hierarchical modeling strategy was used for the modeling of media model package for distillation, as shows in figure 1 . The package has three levels: base level, method level and instantiation level.

The base level model is a partial model named PartialMediaForSepera which all the media models for distillation are extended from. It consists of some common parts that can be shared by all models with the method of two-parameter cubic EoS. The more details are listed as follows.

$>$ Medium constants: media name, names and number of chemical substances, critical proprerties, etc.

$>$ FluidData structure: definition of record type FluidConstants used for storing physicochemical constants of pure substances as show in figure 2. All components' physicochemical constants used in VLE mixture have to comply with this data structure. When a new substance is introduced, the constants are assigned in order of the constants' sequence in figure 2.

$>$ ThermodynamicState: a minimum set of variables of state. It is used for function calling.

> BasePropertiesRecord: common variables could be shared by all media model with all EOS, such as pressure, specific enthalpy of vapor and liquid phase, compressibility factor, etc.

$>$ BaseProperties: a partial model consists of common behavior of all media model with all EOS.

$>$ Type definition: such as "Type CompressibilityFactor $=$ Real(final quantity $=$ "compressibility", $\quad \min =0$, $\max =1) "$. 


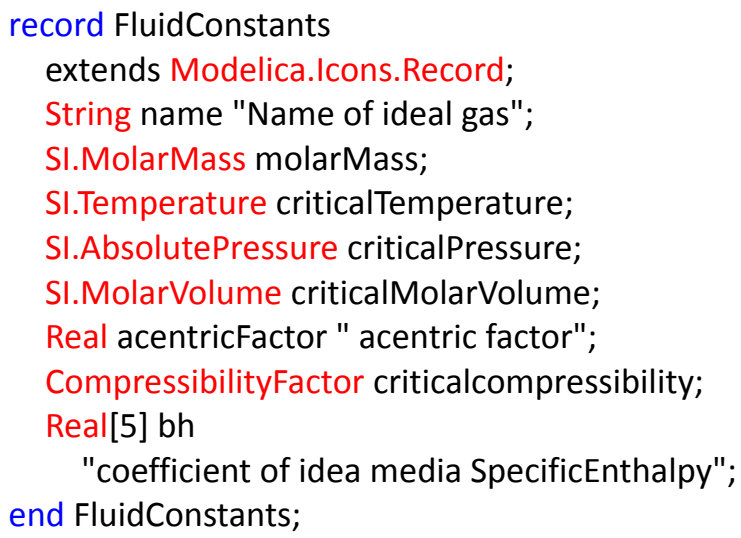

Figure 2 Structure of the FluidConstants

The method level model is extended from the base level model PartialMediaForSepera by adding some special variables and the description according to the different two-parameter cubic EoS methods. For instance, the PR EoS method has the expression of the fugacity coefficient in vapor phase as follow.

$$
\begin{aligned}
\ln \phi_{i}= & \frac{b_{i}}{b_{m}}(z-1)-\ln \left(z-B_{m}\right)- \\
& \frac{A_{m}}{2 \sqrt{2} B_{m}}\left[\frac{2 \sum_{j} x_{j} a_{i j}}{a_{m}}-\frac{b_{i}}{b_{m}}\right] \ln \left(\frac{z+(1+\sqrt{2}) B_{m}}{z+(1-\sqrt{2}) B_{m}}\right)
\end{aligned}
$$

Equation (8) is a special equation which is only suit for the PR EoS but not other. And it should be added to the BaseProperties section when the partial method level media model with the PR method is built by extending the partial base level model, as shows in figure 3.

Finally the case level models are constructed by extending the method level models and adding the components and the mixing rule of mixture. For example, the air model for distillation was built by defining the medium name, components' names and appointing the fluid data of Nitrogen, Oxygen and Argon and the binary interaction coefficient of components as figure 4 .

As discussed above, the reason for introducing the structuralized and hierarchical modeling strategy is that we want to separate the components information of mixture and the mixing rules from VLE predicting method. The same VLE predicting method can be applied to the different mixtures by changing the components information of mixture and mixing rule like the air model showed in figure 4. A user just needs to know the physicochemical constants of components and the mixing rule when the user wants to introduce a new model.

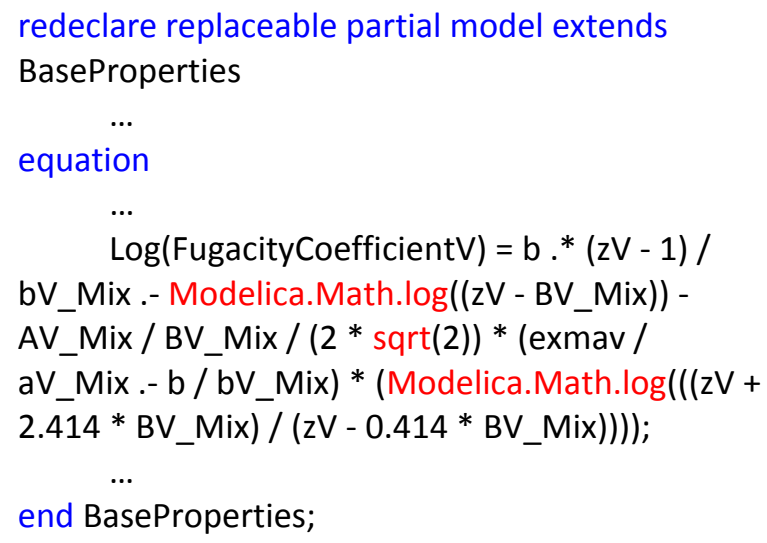

Figure 3 The media model with PR EoS method by extending the base model

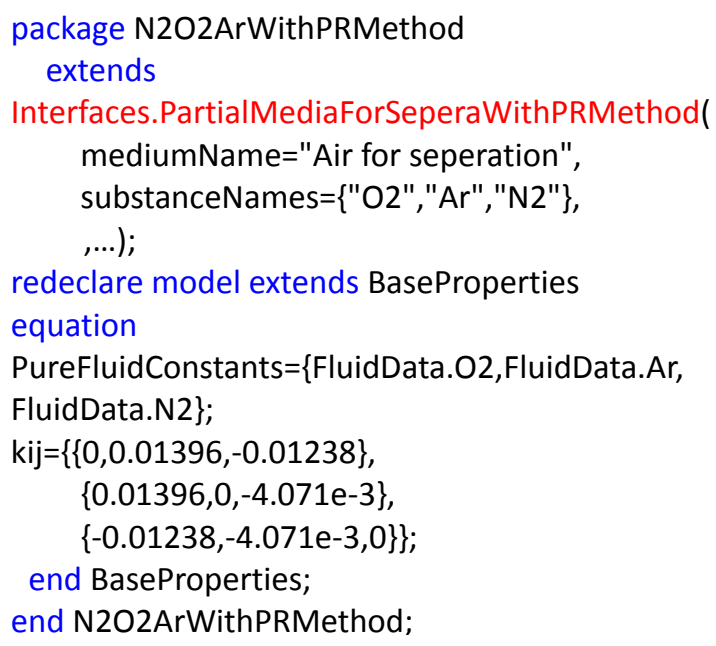

Figure 4 Air model with PR EoS method

\section{Test case}

In order to validate the media model, a steady state air distillation column model was built in Modelica on MWorks based on the assumptions as follows.

$>$ Every column theoretic stage is considered as an adiabatic system.

$>$ In the energy balance the wall material's heat storage is neglected.

$>$ Chemical reactions between Nitrogen, Oxygen and Argon are not considered.

The column contains eighteen theoretic stages and the first of them is the condenser which is at the top of column. The cooled feed air flows into the fifteenth stage. Each theoretic stage obeys the mass and energy balance.

$$
\begin{gathered}
\sum \dot{m}_{i}=0 \\
\sum \dot{m}_{i} h_{i}=0
\end{gathered}
$$


Where, $\dot{m}_{i}$ means the mole flow rate on the inlet and outlet of the theoretic stage. $h_{i}$ the specific enthalpy correspond to the $\dot{m}_{i}$.

The media model used in the column model was the model N2O2ArWithPRMethod mentioned in the
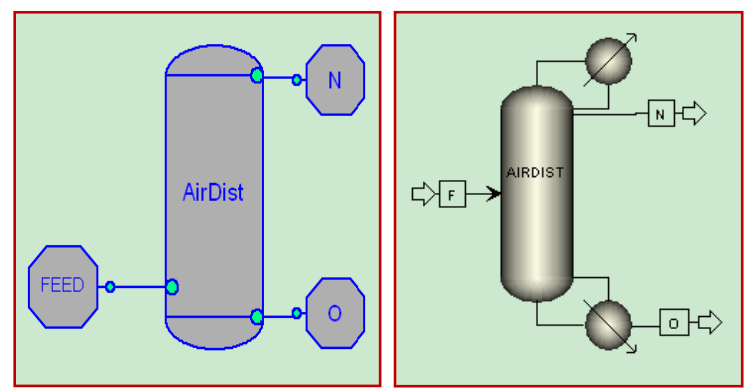

Figure 5 Air distillation column in MWorks (left) and Aspen Plus (right)

third section. The results of this model were validated with calculations simulated with tool Aspen Plus. The figure 5 shows the sketch of air distillation column model in MWorks and Aspen Plus. $\mathbf{F}$ means the cooled feed. $\mathbf{N}$ means nitrogen product. $\mathbf{O}$ means oxygen-enriched liquid air. For the case the following input values and parameters are used.

Table 1 input values and parameters in the model

\begin{tabular}{l|l}
\hline Feed pressure & $6[\mathrm{bar}]$ \\
\hline Feed total flow & $200[\mathrm{kmol} / \mathrm{hour}]$ \\
\hline Feed mole fraction & $0.785[\mathrm{~N} 2], 0.205[\mathrm{O} 2]$, \\
\hline
\end{tabular}

\begin{tabular}{l|l}
\hline & $0.01[\mathrm{Ar}]$ \\
\hline Feed stage & No.15 \\
\hline Stage numbers & 18 \\
\hline Condensation rate & $100 \%$ \\
\hline Condenser pressure & $5[\mathrm{bar}]$ \\
\hline stage pressure drop & $0.01[\mathrm{bar}]$ \\
\hline Distillate rate & $50[\mathrm{kmol} / \mathrm{hour}]$ \\
\hline Reflux & 1.3 \\
\hline
\end{tabular}

The model of air separation tower was simulated with 18 theoretical stages and the input values and parameters used in MWorks and Aspen Plus are listed in table 1 . The physical property model in Aspen Plus is also Peng-Robinson method.

Figure 6 shows the temperature of medium in each stage from the simulation in Aspen Plus and MWorks. The 18th theoretical stage is the oxygen-rich tray and the first stage is the condenser. The maximum difference value of the temperature is $0.201 \mathrm{~K}$ and the mean value of that is $0.1482 \mathrm{~K}$ between the results from MWorks and that from Aspen Plus.Figure 7 demonstrates the values of the mole fraction of components (nitrogen, oxygen and argon) of air in gas phase in each stage from the simulation in MWorks and in Aspen Plus. The maximum difference of mole fraction of nitrogen is $0.1826 \%$ between the results from MWorks and that from Aspen Plus. The mole fraction of oxygen $0.1820 \%$. The mole fraction of argon $0.0049 \%$. The average difference of mole fraction of nitrogen is $0.0778 \%$

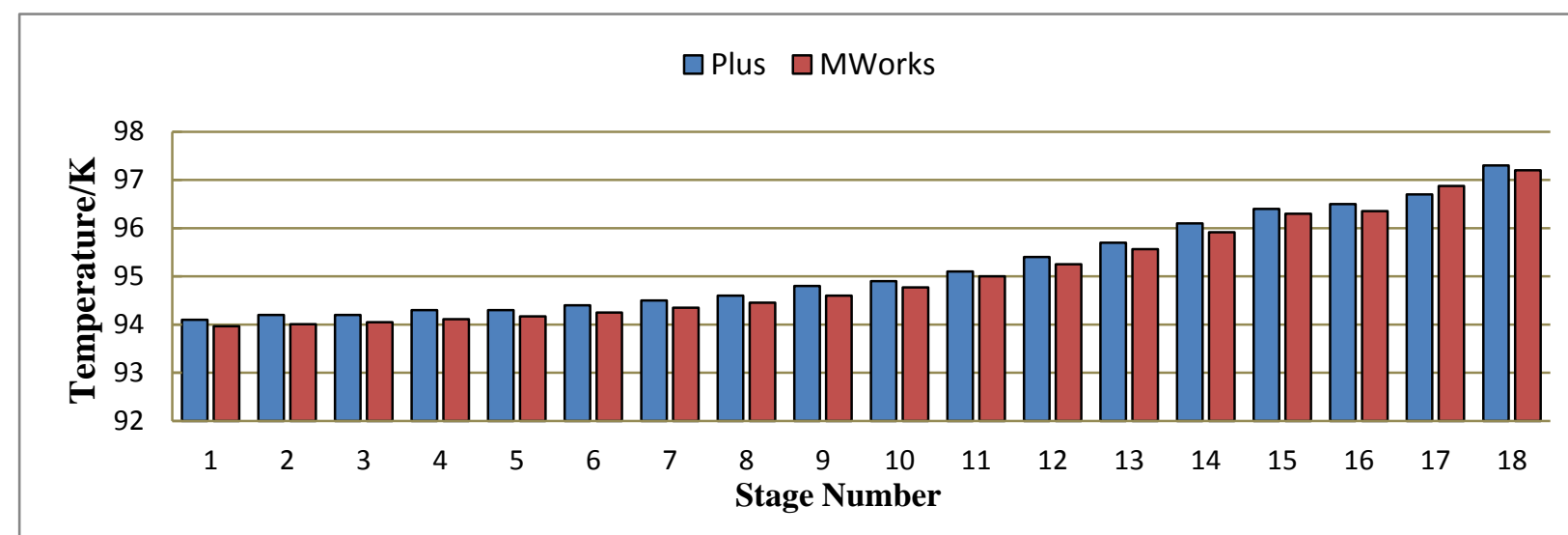

Figure 6 Temperature in each stage 


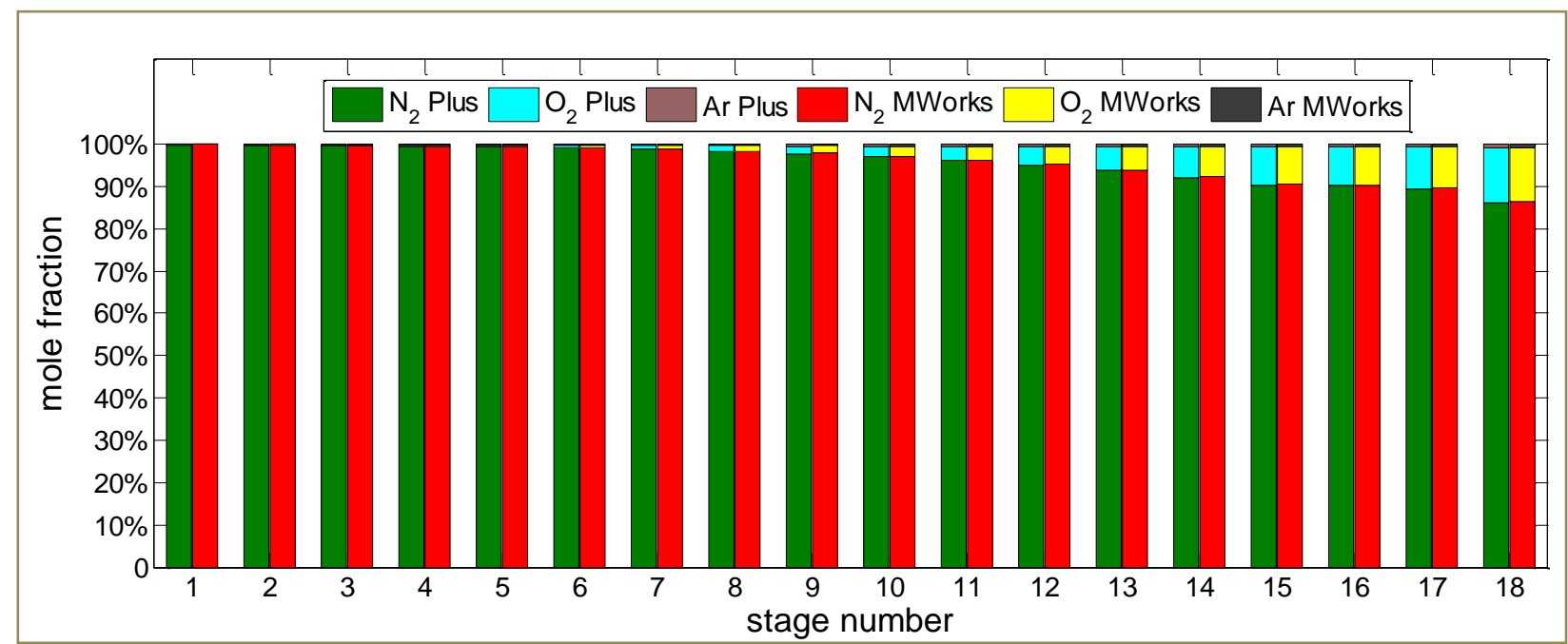

Figure 7 Vapor phase mole fraction profile of air distillation column

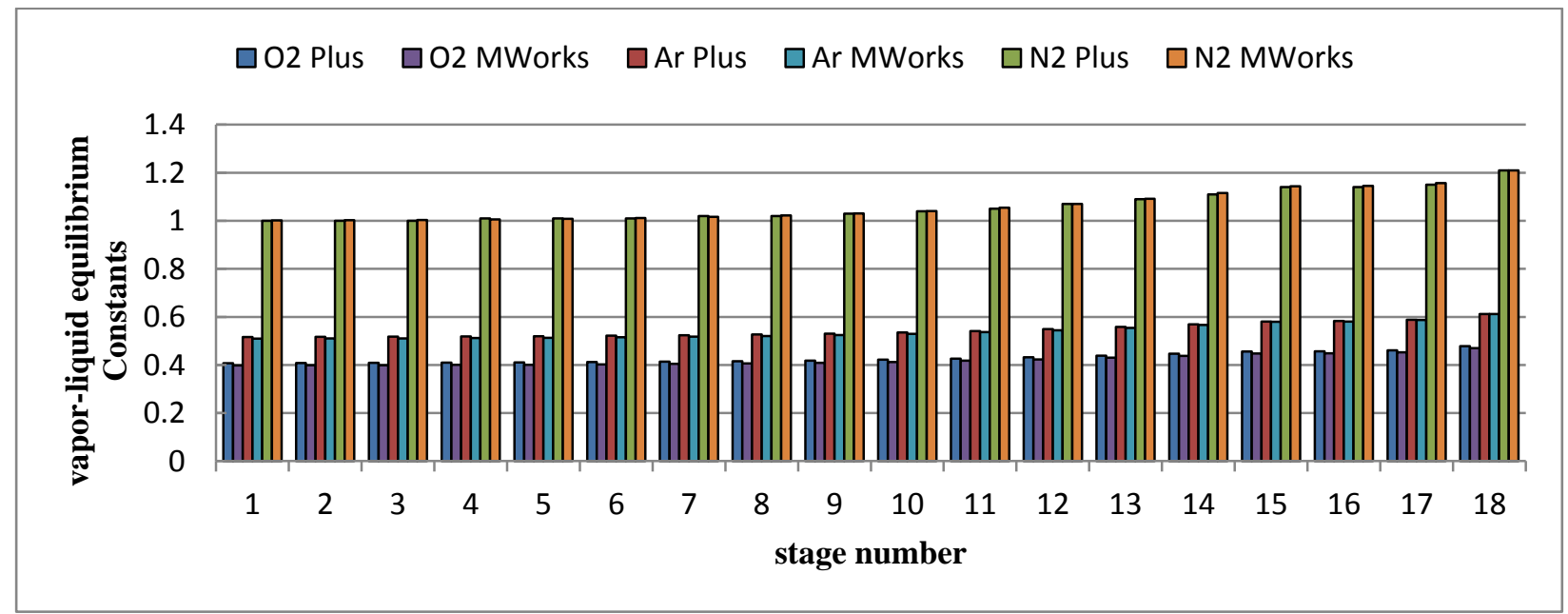

Figure 8 vapor-liquid equilibrium constant profile of air distillation column

between MWorks and Aspen Plus. The mole fraction of oxygen $0.0672 \%$. The mole fraction of argon $0.0025 \%$.

The vapor-Liquid equilibrium constants of substances of mixture simulated in each stage in Aspen Plus and MWorks are showed in Figure 8. The maximum relative difference of the equilibrium constant of nitrogen is $0.6078 \%$ between the results from MWorks and that from Aspen Plus. The equilibrium constant of oxygen $2.4411 \%$. The equilibrium constant of argon $1.4253 \%$. The mean relative difference of the equilibrium constant of nitrogen is $0.2556 \%$ between MWorks and Aspen Plus. The equilibrium constant of oxygen $2.1032 \%$. The equilibrium constant of argon $0.8879 \%$.

Summing up the above, the results obtained in the MWorks model show similar results compared to the Aspen Plus and the media model of air for distillation based on Modelica in MWorks works well.

\section{Conclusions}

Distillation process widely exists in Petroleum refining and chemical industry, et al. The model for vapor-liquid equilibrium predicting is one of the important parts in distillation process. Its computational efficiency and accuracy have a strong impact on the computational efficiency and accuracy of process model. In this paper, a media model package, based on the structuralized and hierarchical modeling strategy, was built for distillation process in order to explore a suitable modeling strategy for media model. An air model with this strategy was used in the air separation column model and the results were validated by the simulation in Aspen Plus. The differences of interested variables' value between the simulation of MWorks and of Aspen Plus are slight and the air model works well.

At present, the method for media model is 
two-parameter cubic EoS and the number of substances is small. The future work is adding the other EoS and activity coefficient method for media model and also substances to make the media package more generally applicable.

\section{Acknowledgments}

The paper was supported by National Nature Science Foundation of China (No. 60704019, No.60874064), Major State Basic Research Development Program of China (No. 2011CB706500).

\section{References}

[1] Modelica Association. Modelica Standard Library, February, 2008. Version 3.0.

[2] Zhou F, Zhang H, Zhu H, et al. Design and Implementation of Animation Post-processor Based on ACIS and HOOPS in MWorks. Proceedings of the 7th International Modelica Conference, Como, Italy, September 20-22, 2009, pp. 663-668.

[3] Reid R C, Prausnitz J M, Poling B E. The properties of gases and liquids. $4^{\text {th }}$ ed. Nem York: McGraw-Hill, 1987.

[4] Harmens. A Cubic Equation of State for the Prediction of O2-Ar-N2 Phase Equilibrium, Cryogenics, v. 17, pp. 519-521, 1977. 